\title{
Urban Open Space's Accessibility Assessment Using Angular Segment to Reinvent as Evacuation Shelter in Historic City
}

\author{
Nattasit Srinurak and Janjira Sukwai
}

\begin{abstract}
This paper using a combination toolset consists of GIS and Angular Segment Analysis in the Space syntax theory to reveal the possibility to reinvent urban open spaces in a historic city regards to its type. The objective of this study is to clarify the relation of auspicious urban open spaces and its urban fabric. Study area in this study is Chiang Mai historic city area situated in Northern part of Thailand. According to urban spatial condition of the city, it has been planned within a strong geometry shape and deform-grid network. The angular segment analysis was used to analyze approaching and angular distance each typology of urban open spaces. The entry points were retrieved by field survey and input to GIS's data. Then, a result of angular segment analysis will overlay with multiple overlay technic. Finally, these overlay's results will be discussed and revealed, urban open space's angular length and spatial data will be classified to created material for reinventing. The result of the study reveals that the relation between religious spaces and its cultural-urban fabric. Urban fabric related to angular length from religious spaces, reflected the cultural-spatial pattern, simultaneously. These determine that religious spaces in Chiang Mai could be reinvented according to its approaching.
\end{abstract}

Index Terms-Open space, emergency shelter, historic city, space syntax.

\section{INTRODUCTION}

Recently, a risk of disaster in the urban area and lack of spatial management are continuously occurring, especially in developing countries [1]. So that the aim of this study is to contribute knowledge into proper policies for spatial improvement of historic city to cope with disaster while maintain irreplaceable historic city character is considering desirable [2], the objective of this study is to analyze and investigate its character of urban fabric to act as evacuation routes due to disaster occurs, focusing on urban morphology and accessibility character.

In present day, misguide rapid development may cause those historic cities prone to disaster risk. Meanwhile, the disaster risk management trend in the 21 st century on preparedness and cycle of disaster risk has placed an importance on disaster risk preparation and its cycle rather

Manuscript received December 30, 2018; revised March 1, 2019. This work was supported in part by the Chiang Mai University.

N. Srinurak, is with the Social Research Institute, Chiang Mai University, 239 Hueykaew, Suthep, Muang, Chiang Mai, Thailand 50200(e-mail: nattasit.s@cmu.ac.th)

J. Sukwai. is with the Graduate School of Regional Design in Art and Economics, Saga University, 1 Honjo-machi, Saga-shi, 840-8502, Japan (e-mail: joanjira@gmail.com) than focusing on recovery strategies [3].

Therefore, to create efficiency risk management, a suitable model must be created by local context consideration, led to the differentiation of solutions in each historic city depend on its social and spatial aspects [4]. Moreover, historic city has vulnerable for experiencing physical problems more than new development cities; the density of buildings, bottleneck situation in circulation within the narrow road, and choices on evacuation routes must be highly considered, combine with its historical meaning [5]. It is an urgency to seek and investigate how the urban morphology of historic city operating in usual basis and its potential to provide evacuation.

In this paper, logic hidden in social dimensions shall be revealed, Open space are usually determined as the gathering center of community combine with cultural aspect during establishment process. However, this complication through a development of the city itself has given effect on the relation of these spaces and its connection to the urban fabric. From this derived issue [6], a reinvention of space is needed to cope with modern problems. In addition, an application from this analysis result will be implied as potential or opportunity issues to reinvent the open space. According to space syntax, Angular Segment analysis is one of the methods that indicate the usage of to-thought traffic in a specific location. Usually, to analyze pathway between $\mathrm{A}$ to $\mathrm{B}$, studies use metric coverage radius as a method. However, according to space syntax - the proven practical theorem for analyzing the relationship between spatial and socio-economic aspects [7] - question in the integrity of conventional vector coverage length method derive.

\section{Methodology}

\section{A. Site of the Study}

Chiang Mai, the ancient capital of the Northern in Thailand, established for 720 years in-between Doi Suthep Mountain and Ping river. The concept of its settlement location was derived from an integrative consideration between religious belief and natural respect, discreetly placing a city in natural rich area and settled its capital in the center as same as other cities in Northern-Thai region at that time. As a natural and cultural prosperous city, Chiang Mai was simultaneously determined to be a capital city and the center of Lan $\mathrm{Na}$ kingdom in ancient time [8] [9].

Chiang Mai historic city continuously developing through time and perceive its own cultural prospect. Since settlement period, its spatial configuration may divide by multi-spatial 
character of the street network with a deformed grid from a delicate shape of symmetry geometry to a free-form natural pedestrian pathway that follows its location of the open spaces. To the fact that multi politic and cultures continually influence the spatial character of Chiang Mai led to the deform grid system road network. As geometric shape of city received and intent to imitate concept of other capital cities established during the same period such as 'Sukhothai' [10] this geometric concept applied to its perimeter shape of city wall and moat that would be useful in wartime, it created area cover 2.56 square kilometers within perfect shape of rectangular perimeter segment. Inner boundary, part of the urban network was intentional designed, as a deform grid hierarchy of street function such as core and cross axis comprise with natural labyrinth sub-network of a residential area.

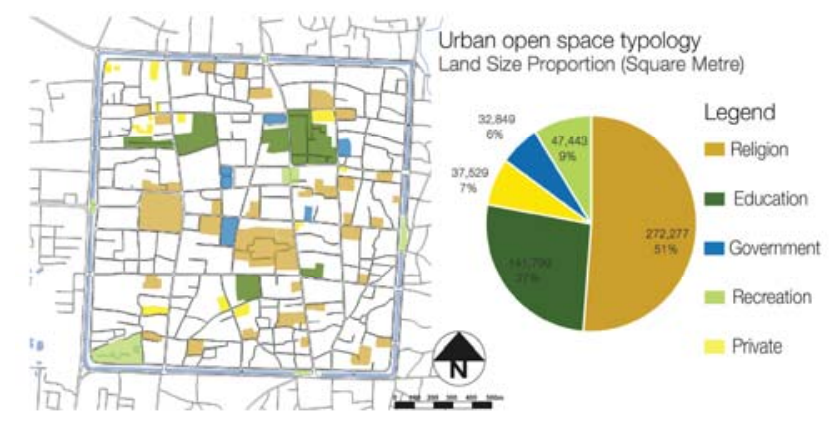

Fig. 1. Urban open space typology and size.

In May 2014, Northern part of Thailand suffered major earthquake disaster with 4-7 Richter, leading more attention on earthquake disaster response/mitigation plan that may had been overlooked [11] [12]. Fortunately, the severe disaster occurred in a rural area which only property damage was found but it raised awareness in earthquake issue, especially in the provinces located on active faults. In addition, disaster vulnerability has raised awareness of earthquake disaster mitigation, especially in historic cities in Northern, Thailand. These cities mostly situated on active faults of seismic activities. Recently, researchers in Thailand have high interest in the Mae Chan fault, which is the most potentially destructive power fault passing through the provinces of Chiang Rai and Chiang Mai. Nowadays, Mae Chan fault remains stable as stress continues to build up. Risk map shows the vulnerability of northern Thailand cities rated by the scale of cities and active faults in the area which Chiang Mai is the most vulnerable city in northern Thailand, [13].

\section{B. Urban Open Space Typology}

To study Chiang Mai's urban character which consists of a building usage and materials, this character was collected and arranged in GIS data format. To evaluate risks on the city, the character of the historic area is one of research materials that needed to be clarified In Chiang Mai's historic city various determinations of urban open spaces were found. In this research, only public usage of urban open spaces in historic area was studied. Type of usage in urban open space consists of religious, education, government, recreation, and private spaces. According to space-used types, spaces are mostly used for religious purpose while following spaces are distributed throughout the area and used as a recreation, education, and private spaces, respectively. However, some of the educational spaces are included as the same area of religious places due to, in the previous time, temples were functioned as the place providing educational function as well as a center of the community to act as a spiritual and cultural related function. As the result, distribution of religious place is occupied and cover throughout the historic area, but educational and government spaces are clustered in in the most center of the city. Moreover, size of religious places is comprised of its attached syntactic properties of the urban network. In addition, most of the religious place is Buddhist temples that established and have developed simultaneously as part of Chiang Mai' morphology. Primary data from this field survey are sorted and arranged in GIS to locate the entry point of each space and will be analyzed in further.
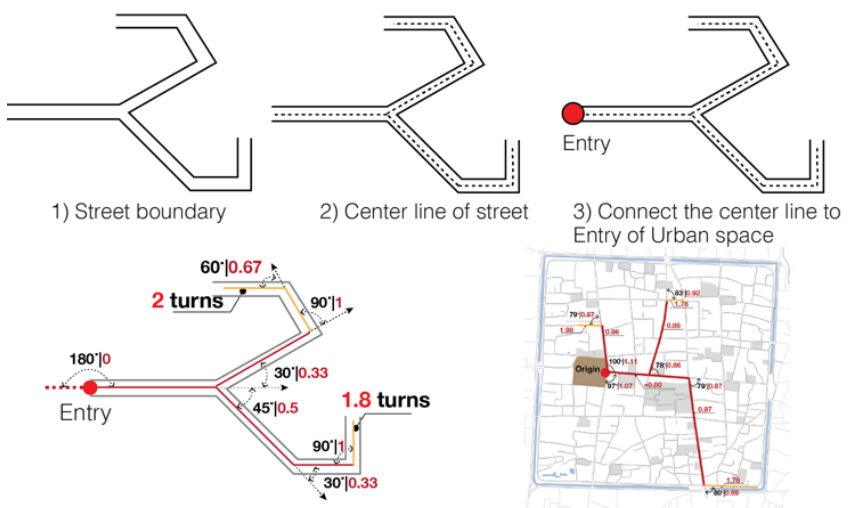

4) Calculate using Angular segment Analysis

\section{5) Mapping to GIS application using overlay technic}

Fig. 2. Procedure of Angular Segment Analysis.

\section{Figures Analysis Method and Procedure}

As individual space will be evaluated it approaching in From-To traffic, space syntax a proven tool 'The Angular segment analysis' was used to evaluate each space's approaching from its entry gates. The Angular Segment Analysis ASA) tool was invented by Hiller and Hanson in 1984 to evaluate spatial morphology in various scales. ASA was introduced and included as on the set of syntactic tools to record weighted of direction change, related to graph theory in mathematic [14]. In this study, a combination tool between ASA and intensity overlay GIS method will be used to evaluate by using the urban open spaces as materials.

In ASA methodology, it found significant relation between this angular turning and natural movement in from-to traffic [15] [16]. The ASA was used to calculate of direction angular change, in this analysis, it was used to evaluate every urban open space according to its entry gate(s) or the origin. Segment lines obtained from the street network then, lines turning will be converted as 90 angles to 1 turn, 45 angles converted as 0.5 turns (see Fig. 2.) Angular turning obtained from this study will be limited by 2 turns (total angular movement $=180$ degrees). In addition, this angular segment analysis will freely analyze in outer rim to avoid edge effect from the boundary of site study [17]. From angular movement result, the length of every segment will be summarized as the angular length from every gate(s) using multiply overlay technic in GIS to combine with the result of each typology of the urban open spaces. 


\section{RESUlT AND DISCUSSION}

ASA result shows the significant relation between location and its urban fabric coverage. Urban open spaces in the historic city were analyzed and reviewed the result according to its typology of usage as follow.

In religious space, the result shows ASA analysis available to clarify feature-related angular length from the open space in the site study. According to the culture-hierarchical location of religious spaces, ASA result shows the relation between temple location and its angular length. It significantly indicated that a distance from the main street (the street with dark red in fig.3.) may highly have an effect on the angular length of an individual temple from its entry gate connection. Every religious space's angular length covered and outreached their surrounding communities with total angular length more than 1,700 meters. These angular length results also signify reasonable walking radius (800 meters) from-to surrounding settlement (see Fig. 3.)

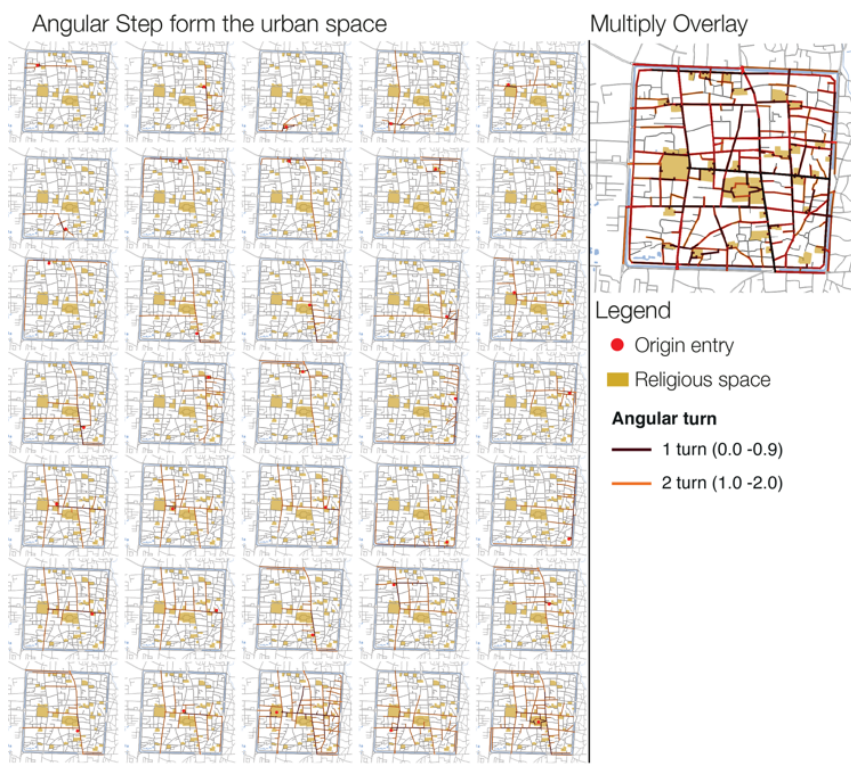

Fig. 3. Religious space angular segment.

In Educational space, the result of ASA shows low profile of angular length in terms of cultural-spatial relation. The location of the spaces is, mostly, located in the northern part of the city and attach to the north-south axis of the street more than the east-west axis of the street. The coverage of angular segment of this type is moderate. The angular segment length clustered in the inner part of the city is more than outreach to outer rim or communities pocket that permeate in the deformed-grid main street. The longest length is 1,140 meters with an average length of 400 meters (see Fig. 4.)

In Government space, the result of ASA shows significantly low angular length that reach to the city's boundary due to its clustering of spaces in the northern part of the city. ASA shows the coverage of the Angular Segment is lacked in the southern part and outer rim of the city. The coverage of angular segment is clustered where the spaces are located, only some of the main north-south axis street is covered and outreach the boundary of the city. Moreover, the coverage is unreached the inner pocket of communities. The longest length has origin from the space in most central, attached to the east-west axis of the city which contain 906 meters from the entry with an average length of 380 meters (see Fig. 5.)

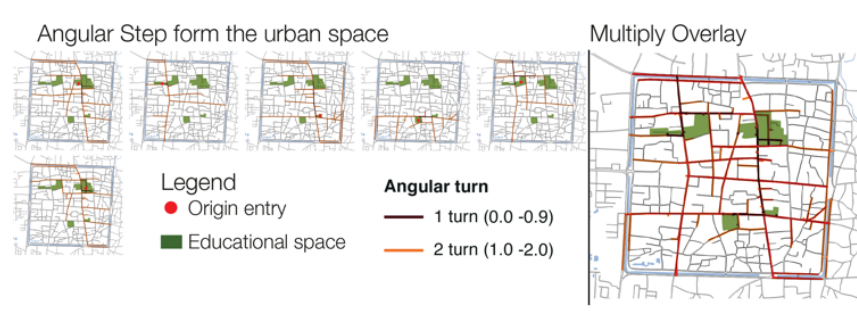

Fig. 4. Educational space angular segment.

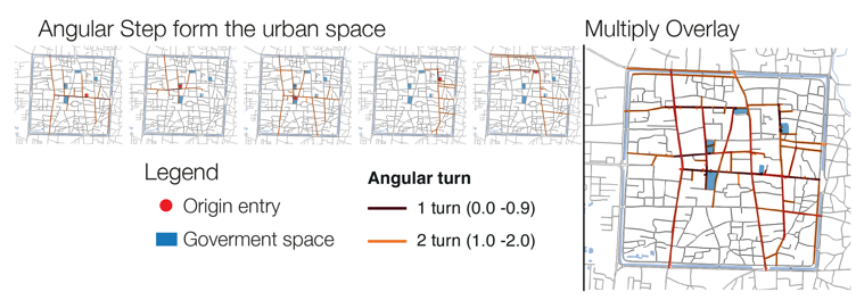

Fig. 5. Government space angular segment.

In recreational space, the Angular segment shows significant high coverage and length. Some of this type of space is traditional space such as the gates of the city. It highly relates to the main street of the city which has an effect on the angular segment coverage of the space even the number of space is quite lower than the other. The location of space is mostly on the outer rim of the city but attached to the end of the main street both in the north-south and the east-west axis. The gates space's angular segment is cover and outreach to the boundary of the city in every direction and also permeate into community pockets. The most outreach coverage comes from the east and the south gate. This result may be explained by the usage of these gate spaces as local markets. Both of the spaces act as urban open space for the commercial area in the past for the east gate and in present-day for the south gate. The south gate has the highest angular length throughout from the north-south axis as 1,600 meters. The recreational space contains average reach of 700 meters. (see Fig. 6.)

In private space, the Angular segment show moderate coverage and length. The space location is distributed throughout the city. It has no pattern of distribution but mostly in the community pocket where labyrinth street is located. The private open space lack in the eastern and southern part of the city and only one of them attach to the main street. The coverage of angular segment is clustered in the central-western part, it outreaches some part of city's boundary but not highly permeate into community pockets. The private space has the highest angular length at 760 meters (lower than a walking distance of 800 meters) and has the average of 300 meters (see Fig. 7.)

To compare the typology of urban open space, in the historic city, the one that related to urban culture such as religious, and commercial spaces seem to have highly angular segment value more than the other types. The highest value of angular segment analysis length is the religious space. This type of space also has the highest location that distributes throughout the historic city. The angular segment 
analysis reveals the firm relation of culture-spatial of the space. It attached to the highest rank of the main street both the north-south axis and the east-west axis. They also permeated their angular length to surrounding community. The size of religious space is the largest, confirmed the appropriate and efficient to be reinvented as an emergency shelter. In addition, the interesting result is derived from recreational space, it contains the efficiency as shown in coverage length of angular segment analysis. The four of city gate open space, boost the angular segment value. According to its location, is attached to all the main streets, led to the high value of angular segment's length. Even though this space contains small size and distribution, but they have the culture-related spatial configuration that led to high angular length efficiency. To affirm the culture-related of angular segment analysis result, the new establishment of open space such as education and government space display the low profile of angular segment results. These types of spaces have low angular segment length, mostly due to their location and entry points. They have no relation to the main street or any surrounding community. The results reveal low permeability of angular segment into the pocket of inner communities. The reason behind this result may come from the later establishment of the spaces which has functioned only on their own but no response to the social scale function of the spaces such a cultural-related space.

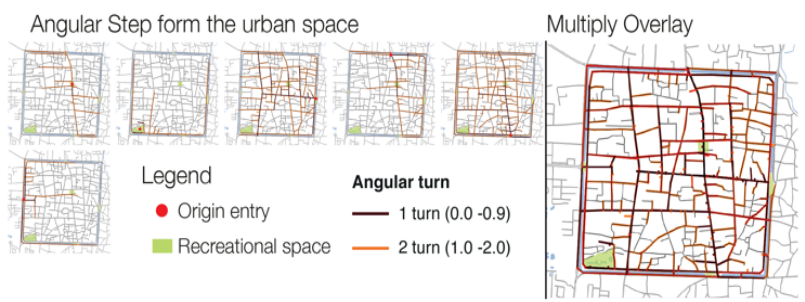

Fig. 6. Recreational space angular segment.

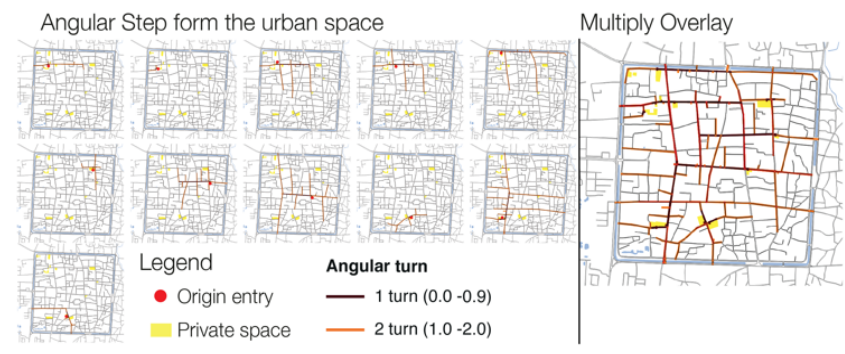

Fig. 7. Private space angular segment.

Additionally, the private space, it may be the unpredictable in near future due to it may contains some constructions and transform it to an occupied space. The private space also has low distribution in the site of study and made it last choice to be reinvented as an emergency shelter.

\section{CONCLUSION}

This paper revealed the underlay cultural-spatial relation of religious space and recreational space with deformed grid street, the historic city in Chiang Mai, Northern Thailand. The Angular Segment Analysis, proven tool in space syntax theory, was used to evaluate the angular length of each individual urban open space. The results from ASA showed highly relation and hierarchy of urban open space based on cultural significance of the space. The higher angular length means that spaces are coped with cultural activities in different levels. Distinctive spaces are also derived and reaffirm a significance of the open space accessibility, these spaces almost cover overall deformed grid network. Groups of religious space were analyzed by its Angular length response directly to its location and link to the urban fabric. There is the urban open space that response to surrounding communities and response to a total historic city as gradually described by this analysis.

To summarize, this study obtained evaluated result that enable supporting data for reinventing cultural urban open space to be an emergency shelter in spatial aspects. Although these spaces contain sensitive cultural-related but have high potentiality to be improved and to gain more advantage in evacuation planning as shelter facility is possible according to its outreach to surrounding communities. Existence and preservation of these spaces could be sustained and adaptable to co-exist with urban fabric and/or forthcoming urban resilience issues.

\section{ACKNOWLEDGMENT}

Surveying in this study has been supported by Saga University and Chiang Mai University. Additionally, authors would like to convey unfathomable gratitude to all informants in Chiang Mai, Thailand.

\section{REFERENCES}

[1] United Nations Human Settlements Programme, Land and Natural Disasters: Guidance for Practitioners, Nairobi: United Nations Human Settlements Programme (UN-HABITAT), 2010.

[2] ICOMOS, "Kyoto declaration 2005 on protection of Cultural Properties, Historic Areas and their settings from loss in disasters," ICOMOS, Kyoto, 2005.

[3] IPCC, Managing the risk of extreme event and disasters to advance climate change adaptation, New York: Cambridge University Press, 2012.

[4] UNESCO, Managing Disaster Risks for World Heritage, Paris: UNESCO World Heritage Centre, 2010.

[5] N. Mishima, N. Miyamoto, Y. Taguchi, and K. Kitakawa, "Analysis of current two-way evacuation routes based on residents' perceptions in a historic preservation area," International Journal of Disaster Risk Reduction, pp. 10-19, 2014.

[6] N. T. Samadhi and N. Tantayanusorn, "Reinventing religious land as urban open space: The case of Kuang in Chiang Mai (Thailand)," Habitat International, vol. 30, no. 4, pp. 886-901, December 2006.

[7] B. Hillier and J. Hanson, The Social Logic of Space, London: Cambridge University Press, 2003.

[8] S. Ongsakul, History of Lanna, Bangkok: Ammarin, 2010.

[9] T. Jarernmuang and D. Apavajaruta, 700 years of Chiang Mai, Bangkok: AkesornSiamKarnpim, 1986.

[10] T. Soraya, Becoming Thai, Bangkok: Chulalongkorn University, 1999.

[11] USGS, "Shakemap," 12 July 2013. [Online]. Available: http://earthquake.usgs.gov/earthquakes/shakemap/. [Accessed 11 December 2015].

[12] MGR online, "Phuket practiced the earthquake-tsunami evacuation (in Thai)," $18 \quad 06 \quad 2017 . \quad$ [Online]. Available: http://www.manager.co.th/Local/ViewNews.aspx?NewsID=95000000 72487. [Accessed 1806 2017].

[13] P. Jarusiri, "Opinion on the likelihood of earthquakes in Thailand," ASTV Manager Daily, 1 May 2012.

[14] N. S. Dalton, Synergy, Intelligibility and Revelation in neighbourhood places, London: University of London, 2010.

[15] B. Hillier, "Studying cities to learn about minds: some possible implication of space syntax for spatial cognition," Environment and Planning B Planning and design, pp. 12-32, 2012.

[16] B. Hillier, "The hidden Geometry of Deformed grids," Environment and Planning B, vol. 26, pp. 169-191, 1999. 
[17] B. Hillier and A. Penn, "Rejoinder to Carlo Ratti," Environmental and Planning B: Planning and Design, pp. 501-511, 2004.

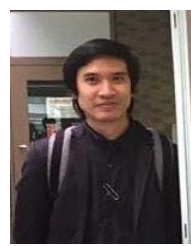

Nattasit Srinurak was born at Chiang Mai, Thailand in 1982. He received his bachelor's degree in architecture from Rajamangala University of Technology Lanna, Thailand in 2008; received his master's degree in urban design from Silpakorn, Thailand in 2011; received his doctor's degree in Engineering (Urban Design) from Saga University, Japan in 2017. The author's major field of study includes disaster reduction in urban design and architecture, urban conservation, and space syntax theory.

For the work experience, he is, 2011-now, the researcher, the Social Research Institute, Chiang Mai University. He conducted several projects as the head researcher including the study of resident behavior in Modification of Building Façade to provide Building Guideline, Pathway to Sustainable University in and Urban planning and Food security. He is interested in Urban design and planning's disaster resilience.

Dr. Nattasit Srinurak is memberships in several societies as follow; Architectural Institute of Japan, Architect Council of Thailand, Thai Urban Designer association. He received some awards as follow; Highest award of research presentation from city planning institute of Japan, Best presentation from 9th international symposium on Lowland Technology, Doctoral degree's scholarship from Japan Government (Monbukagakusho). He also worked for LTI journal as reviewer, and work for Thai Urban Designer Association Lan $\mathrm{Na}$ as a committee.

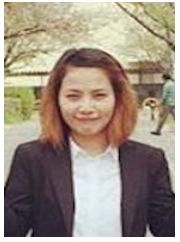

Janjira Sukwai was born at Chiang Mai, Thailand. She is studying in Graduate School of Regional Design in Art and Economics in Saga University, Japan. The author's major field of study includes public participation and urban planning focusing on environmental psychology.

For the work experience, she has experiences working with academic researchers in public participation arrangement. In 2012-2014, she worked as a research assistant of Urban and Environmental Studies at the Social Research Institute, Chiang Mai University.

She conducted several projects as the co-researcher including the study of Resident Behavior in Modification of Building Façade to provide Building Guideline, Pathway to Sustainable University, and Urban Planning and Food Security. She is interested in Public participation, Environmental Psychology, and Urban design and planning, especially public open space. 